\title{
Time Delay-Accelerated Transition of Gene Switch and -Enhanced Stochastic Resonance in a Bistable Gene Regulatory Model
}

\author{
Canjun Wang \\ Nonlinear Research Institute, \\ Baoji University of Arts and Sciences, \\ Baoji 721016, China \\ E-mail: cjwangbj@126.com
}

\author{
Ming Yi \\ Wuhan Institute of Physics and Mathematics, \\ Chinese Academy of Sciences, \\ Wuhan 430071, China \\ E-mail: yiming@wipm.ac.cn
}

\author{
Keli Yang \\ Nonlinear Research Institute, \\ Baoji University of Arts and Sciences, \\ Baoji 721016, China \\ E-mail:yklbj@163.com
}

\begin{abstract}
The roles of time delay on gene switch and stochastic resonance are systematically explored based on a famous gene transcriptional regulatory model with noises. Our theoretical results show that the time delay can induce the switch, i.e., the TF-A monomer concentration shifts from the high concentration state to the low concentration state ("on" $\rightarrow$ "off"), and can further accelerate the transition from "on" to"off". Moreover, it is found that the stochastic resonance can be enhanced by the time delay and the correlated noise intensity. However, the additive noise original from the synthesis rate restrains the stochastic resonance. It is very interesting that the resonance bi-peaks structure appears for the large value of the additive noise intensity. The theoretical results by using small-delay timeapproximation approach are consistent well with our numerical simulation.
\end{abstract}

\section{INTRODUCTION}

In recent years, a plenty of researches show that noises play a positive role in many fields. Many novel phenomena are found, such as, noise induced transition[1], [2], [3], reentrance phenomena[4], [5], stochastic resonance[6], [7], noise enhance stability[8], [9], current reveal[10], [11], [12], and so on. On the other hand, in many cases, the delay reflects transmission times related to the transport of matter, energy, and information through the system. Understanding the behavior of time-delayed dynamical systems is a first step in improving the knowledge of memory in general, whose analysis is especially important in medicine, biology and control theory. Recently, the combined effects of noises and time delays have been the subject of increased interest. In the field of pure statistical physics, the bistable systems with noise and time delay simultaneous have been mainly investigated in detail[13], [14], [15]. Brownian motor with time-delayed feedback is studied by $\mathrm{Wu}[16]$. The effect of time delay on feedback control of a flashing ratchet has been also investigated [17]. The integration of stochastic noise and time delay completely suppresses the population explosion in a mutualism[18]. Effects of time delays and noises in competitive systems have been investigated[19]. These results implicated that the combination of noise and time delay could provide an efficient tool for understanding real systems.
Regulation of gene expression by signals outside and inside the cell plays important roles in many biological processes. As the basic principles of genetic regulation have been characterized, it has become increasingly evident that nonlinear interactions, positive and negative feedback within signaling pathways, time delays, protein oligomerization, and crosstalk between different pathways need to be considered to fully understand genetic regulation[20], [21], [22], [23], [24], [25], [26]. Smolen et al. have introduced a simple genetic regulatory model that incorporate known features of genetic regulatory using an explicitly mathematical dynamic systems approach[20], [21]. The simplest model manifested multiple stable steady states, and brief perturbations could switch the model between these states. Moreover, the effects of macromolecular transport and stochastic fluctuations on dynamics of genetic regulatory systems are investigated. Liu [23]et al. have studied the effects of the correlation between the noise of the decomposed rate $k_{d}$ and the noise of the synthesis rate $R_{\text {bas }}$. They found that a successive switch process (i.e., "on" $\rightarrow$ "off" $\rightarrow$ "on", which we call the reentrance transition or twice switch) occurs with increasing the noise intensities, and a critical noise intensity exists at which the mean first passage time of the switch process is the largest. The effect of the color cross-correlated on the switch is investigated[24]. Wang[25] et al. also have investigated the effects of delay time, which is the time required for movement of TF-A protein to the nucleus. Their results showed that the delay time restrains the transition from the low concentration state to the high concentration state. However, these studies only consider single noise source, in particular, the delay-induced switch-like behaviors has not been explored yet. In addition, in this case, the delay time appears in both deterministic and fluctuating forces simultaneously, hence it is very difficult to study from a view of theoretical analysis.

Stochastic resonance (SR), which was originally discovered by Benzi and Nicolis[27], [28] in the context of modeling the switch of the Earth's climate between ice ages and periods of relative warmth, is an important aspect in many scientific 
fields, which has been investigated extensively due to its potential applications from both the theoretical and experimental points of view. SR is a common case and generic enough to be observable in a large variety of nonlinear dynamical systems, including the occurrence of SR in physical systems, biological system, ecological system, laser system, etc. In the biophysics field, the study of SR phenomenon has turned into a forward subject. The SR phenomenon and its applications were extensively found, such as, noise enhancement of information transfer in crayfish mechanoreceptors by SR [7]. SR can be used as a measuring tool to quantify the ability of the human brain to interpret noise contaminated visual patterns [29] and SR appears in an anti-tumor system modulated by a seasonal external field [30]. Oscillation and noise determine signal transduction in shark multimodal sensory cells[31]. The gene expression can be regulated by signals from outside and within the cell. Thereby, in the gene transcriptional regulatory process, the external environmental factors, such as, the electromagnetic field on the earth, the solar terms, seasonal variation, are the common features. This means that the transcriptional regulatory of gene should have a periodic form. In this case, the bistability, noise and the signal exist simultaneously, so the combined effects of noises and delay time on the SR should be investigated.

We would like to emphasize that the combined effects of noise and time delay on dynamical behaviors of gene regulatory network is rarely investigated. Our investigation is a significant try forward understanding the basic mechanisms of the delay induced gene switch and stochastic resonance in realistic yet complex organisms from a view of theory, and will motivate the further experimental research for gene network.

\section{MODEL}

\section{A. Deterministic gene regulatory model}

To examine the capability of genetic regulatory systems for complex dynamic activity, Smolen et al.[20] have developed simple kinetic models that incorporate known features of these systems. These features include autoregulation and stimulus-dependent phosphorylation of transcription factors (TFs), dimerization of TFs, crosstalk, and feedback. The simplest kinetic model of genetic regulation can be described by Fig. 1. A single TF-A is considered as part of a pathway mediating a cellular response to a stimulus. The TF forms a homodimer that can bind to responsive elements.(TF-REs). The TF-A gene incorporates a TF-RE, and when homodimers bind to this element, TF-A transcription is increased. Binding to the TF-REs is independent of dimer phosphorylation. Only phosphorylated dimers can activate transcription. The fraction of dimers phosphorylated is dependent on the activity of kinases and phosphatases whose activity can be regulated by external signals. Thus, this model incorporates both signalactivated transcription and positive feedback on the rate of TF synthesis. It is assumed that the transcription rate saturates with TF-A dimer concentration to maximal rate $k_{f}$, which is proportional to TF-A phosphorylation. At negligible dimmer

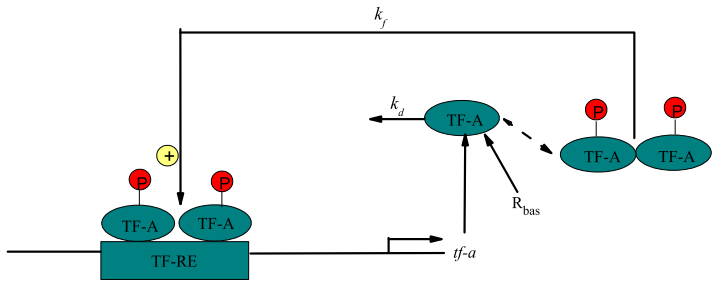

Fig. 1. Model of genetic regulation with a positive autoregulatory feedback loop. The transcription factor activator (TF-A) activates transcription with a maximal rate $k_{f}$ when phosphorylated (P) and binds as a dimer to specific responsive-element DNA sequences (TF-REs). TF-A is decomposed with rate $k_{d}$ and synthesized with rate $R_{b a s}$

concentration, the synthesis rate is $R_{\text {bas }}$. TF-A is eliminated with a rate constant $k_{d}$, binding processes are considered comparatively rapid, so the concentration of dimmer is proportional to the square of TF-A monomer concentration $x$. These simplifications give a model with a single ordinary differential equation for the concentration of the TF-A:

$$
\frac{d x(t)}{d t}=\frac{k_{f} x^{2}(t)}{x^{2}(t)+K_{d}}-k_{d} x(t)+R_{b a s}
$$

where $K_{d}$ is the dissociation concentration of the TF-A dimer from TF-REs. Under the following condition of parameters:

$$
\begin{aligned}
& {\left[-\left(\frac{k_{f}+R_{\text {bas }}}{3 k_{d}}\right)^{3}+\frac{K_{d}\left(k_{f}+R_{\text {bas }}\right)}{6 k_{d}}-\frac{K_{d} R_{\text {bas }}}{2 k_{d}}\right]^{2}+} \\
& {\left[\frac{K_{d}}{3}-\left(\frac{k_{f}+R_{b a s}}{3 k_{d}}\right)^{2}\right]^{3}<0 .}
\end{aligned}
$$

The potential function corresponding to Eq.(1) is

$$
U_{0}(x)=k_{f} \sqrt{K_{d}} \arctan \left(\frac{x}{\sqrt{K_{d}}}\right)+\frac{k_{d}}{2} x^{2}-\left(R_{b a s}+k_{f}\right) x .
$$

Two stable steady states are presented as $x_{+}=2 \sqrt{-p / 3} \cos (\theta)+\left(R_{\text {bas }}+k_{f}\right) /\left(3 k_{d}\right)$ and $x_{-}=2 \sqrt{-p / 3} \cos (\theta+2 \pi / 3)+\left(R_{b a s}+k_{f}\right) /\left(3 k_{d}\right)$. One unstable steady state is $x_{u}=2 \sqrt{-p / 3} \cos (\theta+4 \pi / 3)+$ $\left(R_{\text {bas }}+k_{f}\right) /\left(3 k_{d}\right)$, where $p=K_{d}-\left[\left(R_{\text {bas }}+k_{f}\right) / k_{d}\right]^{2} / 3$, $q=K_{d}\left(k_{f}-2 R_{\text {bas }}\right) /\left(3 k_{d}\right)-2\left[\left(R_{\text {bas }}+k_{f}\right) /\left(3 k_{d}\right)\right]^{3}$ and $\theta=\arccos \left(-q /\left(2 \sqrt{-p^{3} / 27}\right) / 3\right.$.

An interesting aspect of the model is that, based on the different initial conditions, the concentration of TF-A can be one of the two stable steady states. So it is a bistable system for the certain values of $k_{f}\left(5.45<k_{f}<6.68\right)$ (see Fig. 2). Bistable systems are a kind of important system in biological systems. In this article, our works are employed in the bistable region. When the parameter values are $k_{f}=6, K_{d}=10, k_{d}=1$ and $R_{\text {bas }}=0.4$, the stable steady states are $x_{-} \approx 0.62685$ and $x_{+} \approx 4.28343$, and the unstable steady state is $x_{u} \approx 1.48971$ as shown in Fig.3[23]. 


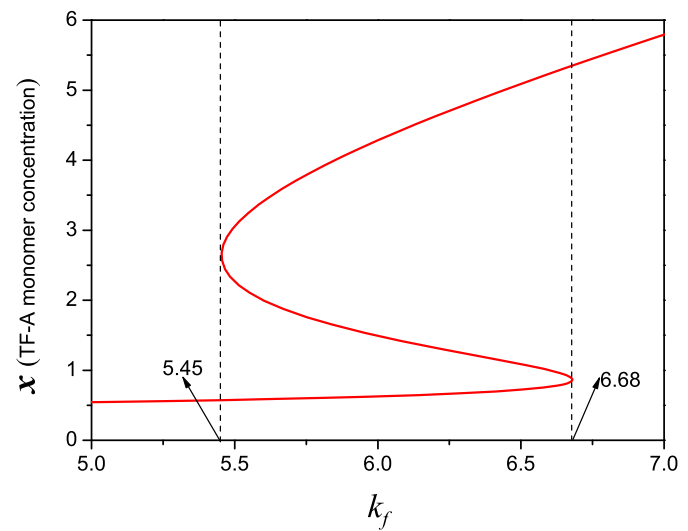

Fig. 2. Bifurcation plots for the steady state of TF-A on the control parameter of transcription rate $k_{f}$. The system in the region (i.e., $5.45<k_{f}<6.68$ ) exhibits multistability. The other parameters are fixed as dissociation constant of TF-A dimer from TF-REs $K_{d}=10$, the degrade constant $k_{d}=1$, and the basal rate of TF-A synthesis $R_{b a s}=0.4$.

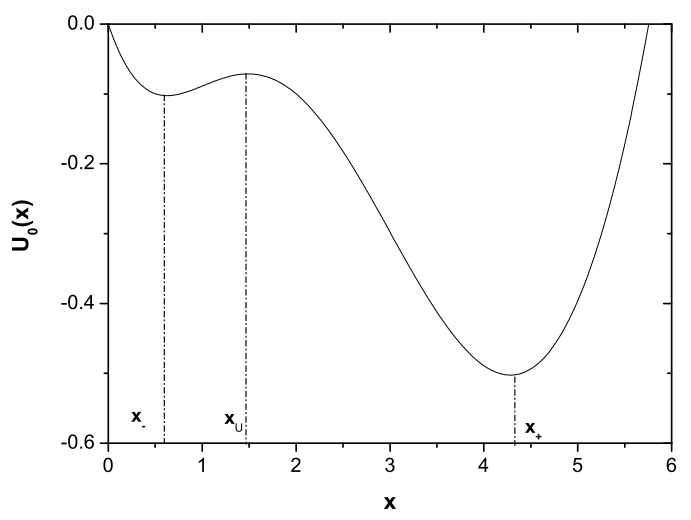

Fig. 3. The bistable potential of Eq.(3). The parameter values are $k_{f}=6$, $K_{d}=10, k_{d}=1$, and $R_{b a s}=0.4$. The stable steady states are $x_{-}$and $x_{+}$, and the unstable steady state is $x_{u}$.

\section{B. Stochastic model with correlated noise and time delay}

Cells are intrinsically noisy biochemical reactors: low reactant numbers can lead to significant statical fluctuations in molecule numbers and reaction rates[32]. It has been found that the stability against fluctuations is essential for the gene regulatory cascade controlling cell differentiation in a developing embryo [33]. Moreover, these fluctuations are intrinsic: they are determined by structure, reaction rates, and species concentrations of the underlying biochemical networks. So we should not only consider deterministic model. Recently, some experiments also showed that $R_{b a s}$ and $k_{d}$ are affected by the biochemical reactions, mutations, and the concentrations of other proteins are also fluctuant[34]. Therefore, it is reasonable to consider the fluctuation effects on the gene transcriptional regulatory model. We consider the fluctuations

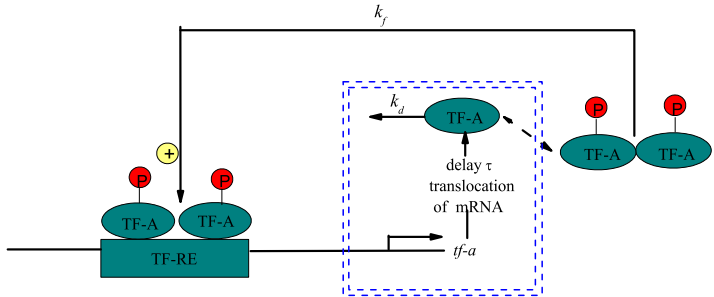

Fig. 4. Model of genetic regulation with a positive autoregulatory feedback loop and delay time.

both on the synthesis rate $R_{\text {bas }}$ and the rate constant $k_{d}$. Namely, $R_{\text {bas }} \rightarrow R_{\text {bas }}+\eta(t)$ and $k_{d} \rightarrow k_{d}+\xi(t)$. The two independent noise $\xi(t)$ and $\eta(t)$ may have a common source, thereby, the correlation between them should be taken into our model. The stochastic differential equation (Langevin equation) corresponding to this bistable gene model is given:

$$
\frac{d x(t)}{d t}=\frac{k_{f} x^{2}(t)}{x^{2}(t)+K_{d}}-\left(k_{d}+\xi(t)\right) x(t)+R_{b a s}+\eta(t)
$$

where $\xi(t)$ and $\eta(t)$ are the Gaussian white noise with the following statistical properties:

$$
\begin{gathered}
\langle\xi(t)\rangle=\langle\eta(t)\rangle=0, \\
\left\langle\xi(t) \xi\left(t^{\prime}\right)\right\rangle=2 D \delta\left(t-t^{\prime}\right), \\
\left\langle\eta(t) \eta\left(t^{\prime}\right)\right\rangle=2 \alpha \delta\left(t-t^{\prime}\right), \\
\left\langle\xi(t) \eta\left(t^{\prime}\right)\right\rangle=\left\langle\eta(t) \xi\left(t^{\prime}\right)\right\rangle=2 \lambda \sqrt{\alpha D} \delta\left(t-t^{\prime}\right) .
\end{gathered}
$$

where $D$ and $\alpha$ denote the multiplicative and additive noise intensities, respectively. And $\lambda$ represents the coupling strength between the two noise terms (i.e., correlated intensity).

Smolen et al.[20], [21] have considered that the gene transcriptional regulatory binding processes are comparatively rapid, and would probably not be reasonable for overall cellular nuclear concentration of TF-A, because the equilibration time would be on the order of the degradation time for TFA protein. However, a short time scale for equilibration is more likely for nuclear concentration of TF-A. This is because the rate constants $k_{f}$ and $k_{d}$ include implicitly entrance and exit of TF-A protein from the relatively small nuclear volume and are thus larger than those governing the dynamics of overall cellular concentration of TF-A. Therefore, the time delay should be considered in this model. This delay appears between any change in the level of nuclear TF-A and the appearance in the nucleus of TF-A synthesized in response to that change. The simplest kinetic model of genetic regulation with time delay can be described by Fig.4.

In order to more exactly predict the dynamics of the genetic regulation model, it is necessary to consider macromolecular transport in these biochemical reactions. Transport can be diffusive or active, and in some cases a time delay may suffice to model active transport. We consider the time delay $\tau$, which 
is the time taken for the transcription of $t f-a$ mRNA and its movement to translation. This delay can affect the TF-A monomer concentration $x(t)$. Therefore, $\left(k_{d}+\xi(t)\right) x(t)$ can be written as $\left(k_{d}+\xi(t)\right) x(t-\tau)$, and Eq. (4) can be rewritten:

$\frac{d x(t)}{d t}=\frac{k_{f} x^{2}(t)}{x^{2}(t)+K_{d}}-k_{d} x(t-\tau)+R_{\text {bas }}-x(t-\tau) \xi(t)+\eta(t)$,

where the $\tau$ (time delay) previous to the time when $d x / d t$ is computed. In addition, only small time delay is investigated in our article so that the theoretical approximation methods below are applicable in our model.

\section{DYNAMICAL CHARACTERS}

\section{A. Steady-state probability distribution}

The small delay time approximation of the probability density approach is employed [35], [36]. Substituting $x_{\tau}$ for $x(t-\tau)$ in Eq.(9), we obtain

$$
\frac{d x(t)}{d t}=h_{e f f}(x(t))+g_{e f f}(x(t)) \xi(t)+\eta(t),
$$

where

$$
\begin{aligned}
& h_{e f f}(x)= \int_{\substack{-\infty \\
\times}}^{+\infty}\left(\frac{k_{f} x^{2}}{x^{2}+K_{d}}-k_{d} x_{\tau}+R_{\text {bas }}\right) \\
&=(1+\tau)\left(\frac{k_{f} x^{2}}{x^{2}+K_{d}}-k_{d} x+R_{\text {bas }}\right) . \\
& g_{\text {eff }}(x)=\int_{-\infty}^{+\infty}\left(-x_{\tau}\right) P_{s}\left(x_{\tau}, t-\tau ; x, t\right) d x_{\tau}=-(1+\tau) x .
\end{aligned}
$$

In Eq.(11-12), $P_{d}\left(x_{\tau}, t-\tau ; x, t\right)$ and $P_{s}\left(x_{\tau}, t-\tau ; x, t\right)$ denote

the conditional distributions of $x(t)$ in the determinate part and stochastic part, respectively, which are given by[37]

$$
\begin{aligned}
P_{d}\left(x_{\tau}, t-\tau ; x, t\right)= & \sqrt{\frac{1}{2 \pi G^{2}(x, x) \tau}} \\
& \times \exp \left(-\frac{\left[x_{\tau}-(x+h(x, x) \tau)\right]^{2}}{2 G^{2}(x, x) \tau}\right), \\
P_{s}\left(x_{\tau}, t-\tau ; x, t\right)= & \sqrt{\frac{1}{2 \pi G^{2}(x, x) \tau}} \\
& \times \exp \left(-\frac{\left[x_{\tau}-(x+g(x, x) \tau)\right]^{2}}{2 G^{2}(x, x) \tau}\right)
\end{aligned}
$$

where $h(x, x)=\frac{k_{f} x^{2}}{x^{2}(t)+K_{d}}-k_{d} x+R_{\text {bas }}, g(x, x)=-x$, $G^{2}(x, x)=D x^{2}-2 \lambda \sqrt{D \alpha} x+\alpha$. Thus, the stochastic delayed differential equation can be approximately reduced to the ordinary stochastic equation. The non-Markovian process induced by the time delay can be transformed to Markovian process. Meanwhile, Eq.(10) can be equivalently transformed into a stochastic differential equation[2]

$$
\frac{d x(t)}{d t}=h_{e f f}(x(t))+G_{e f f}(x) \varepsilon(t)
$$

with

$$
\left\langle\varepsilon(t) \varepsilon\left(t^{\prime}\right)\right\rangle=2 \delta\left(t-t^{\prime}\right),
$$

$$
\begin{aligned}
G_{e f f}(x) & =\sqrt{D g_{e f f}(x)^{2}-2 \lambda \sqrt{D \alpha} g_{e f f}(x)+\alpha} \\
& =\sqrt{D(1+\tau)^{2} x^{2}-2 \lambda \sqrt{D \alpha}(1+\tau) x+\alpha}
\end{aligned}
$$

In the steady-state regime (given by Eq.(2)) and under the constraint $x>0$ (the TF-A monomer concentration $x(t)$ is all higher than zero), the approximate delay Fokker-Planck equation corresponding to Eq.(15) is derived as

$$
\frac{\partial}{\partial t} P(x, t)=-\frac{\partial}{\partial x} A(x) P(x, t)+\frac{\partial^{2}}{\partial x^{2}} B(x) P(x, t) .
$$

where

$$
\begin{gathered}
A(x)=h_{e f f}(x)+G_{e f f} \frac{d G_{e f f}(x)}{d x}, \\
B(x)=G_{e f f}^{2}(x) .
\end{gathered}
$$

The stationary probability distribution (SPD) corresponding to Eq. (18) is obtained

$$
\begin{aligned}
P_{s t}(x) & =\frac{N}{G_{e f f}} \exp \int_{0}^{x} \frac{h_{e f f}\left(x^{\prime}\right)}{B\left(x^{\prime}\right)} d x^{\prime}, \\
& =\frac{N}{G_{e f f}} \exp [\Phi(x)],
\end{aligned}
$$

where $N$ is a normalization constant, and $\Phi(x)$ is the generalized potential function following

$$
\begin{aligned}
\Phi(x)= & \frac{1}{\gamma_{0}}\left[\gamma_{1} \ln \left(x^{2}+K_{d}\right)+\gamma_{2} \arctan \left(\frac{x}{\sqrt{K_{d}}}\right)\right. \\
& +\gamma_{3} \ln \left(D n^{2} x^{2}-2 m n x+\alpha\right) \\
& \left.+\frac{\gamma_{4}}{\sqrt{D \alpha n^{2}-m^{2} n^{2}}} \arctan \left(\frac{D n^{2} x-m n}{\sqrt{D \alpha n^{2}-m^{2} n^{2}}}\right)\right],
\end{aligned}
$$

where

$$
\begin{aligned}
n= & 1+\tau \\
m= & \lambda \sqrt{D \alpha} \\
\gamma_{0}= & 4 m^{2} K_{d} n^{2}-2 \alpha D n^{2} K_{d}+K_{d}^{2} D^{2} n^{4}+\alpha^{2}, \\
\gamma_{1}= & -K_{d} k_{f} n^{2} m \\
\gamma_{2}= & K_{d}^{3 / 2} k_{f} D n^{3}-n \sqrt{K_{d}} k_{f}, \\
\gamma_{3}= & n^{2} m K_{d} k_{f}-\frac{k_{d} \alpha^{2}}{2 D n}-\frac{2 n m^{2} K_{d} k_{d}}{D} \\
& +n \alpha k_{d} K_{d}-\frac{D n^{3} K_{d}^{2} k_{d}}{2}, \\
\gamma_{4}= & -\alpha K_{d} D n^{3} k_{f}+n \alpha^{2} k_{f}+4 n^{3} m^{2} R_{b a s} k_{d} \\
& -2 D n^{3} R_{b a s} K_{d} \alpha+D^{2} n^{5} R_{b a s} K_{d}^{2}+n R_{b a s} \alpha^{2} \\
& +2 n^{3} m^{2} K_{d} k_{f}-m k_{d} \alpha^{2}-4 n^{2} m^{3} K_{d} k_{d} \\
& +2 n^{2} m \alpha k_{d} K_{d}-n^{4} m D K_{d}^{2} k_{d} .
\end{aligned}
$$

In the bistable region, the time course of TF-A monomer concentration $x(t)$ and the probability distribution are plotted by directly simulating the stochastic differential equation (9) 


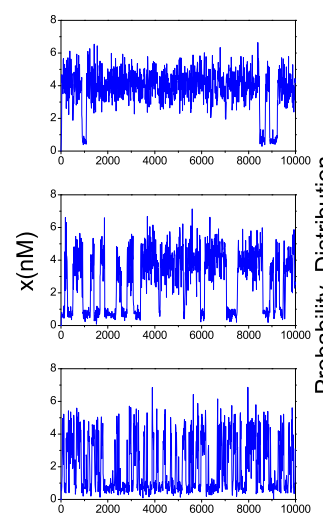

time(min)

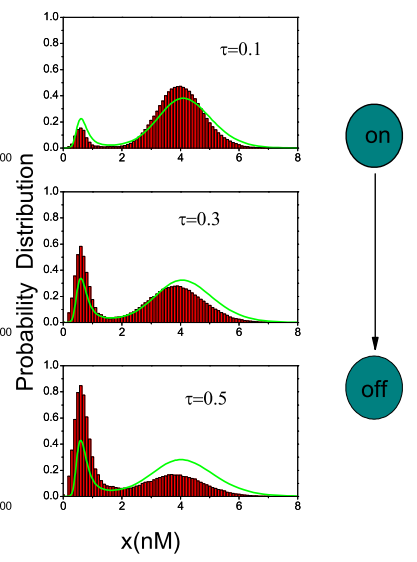

Fig. 5. Sample paths and probability distribution of $x(t)$ for different delay time $\tau$. From top to bottom $\tau=0.1,0.3$, and 0.5. $\alpha=0.01, \mathrm{D}=0.15$ and $\lambda=0.3$. The green curve in right is the SPD by using of Eq. (21). The other parameter values are the same as those in Fig.3.

and by using the theoretical formula (21) for different delay time, as shown in Fig. 5, respectively. From Fig.5, it is clear that the TF-A monomer concentration $x$ shifts from the high concentration state to the low concentration state with increasing the delay time $\tau$. If we regard the low concentration state as the "off" state and the high concentration state as the "on" state, the above result indicates that a switch process can be induced by the delay time. Figure 5 shows that the TF-A monomer concentration $x$ concentrates on the high concentration state when the delay time $\tau$ is small, that is, we begin the switch in the "on" position by tuning the delay time to a very low value. However, increasing the delay time causes the low concentration state to become populated. It means that the concentration of TF-A monomer decreases, and a flipping of the switch to the "off" position occurs. Therefore, delay time can be used as a control parameter for the switch process in the genetic regulatory system. The agreement between the theoretical and the numerical results indicates that the approximation method seems to work quite well for small delay time.

\section{B. Mean value}

In order to quantitatively investigate the stationary properties of the system, we introduce the moments of the variable $x$ as

$$
\left\langle x^{n}\right\rangle_{s t}=\int_{0}^{+\infty} x^{n} P_{s t}(x) d x .
$$

The mean of the state variable $x$ is

$$
\langle x\rangle_{s t}=\int_{0}^{+\infty} x P_{s t}(x) d x .
$$

The theoretical and the numerical simulation results of $\langle x\rangle_{s t}$ as a function of $\tau$ is plotted on Fig.6. Figure 6 shows that the

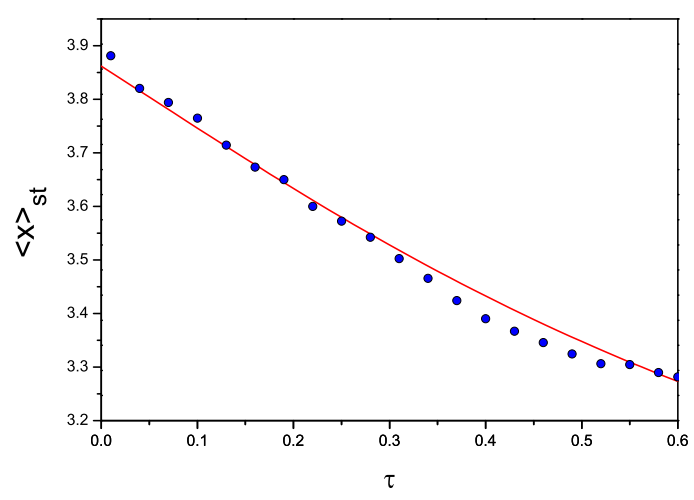

Fig. 6. $\langle x\rangle_{\text {st }}$ is plotted as a function of delay time $\tau$. $\alpha=0.01, D=0.015$ and $\lambda=0.3$. The other parameter values are the same as those in Fig. 3. The red sold line represents the theoretical results, and the blue dot line represents the numerical simulation results.

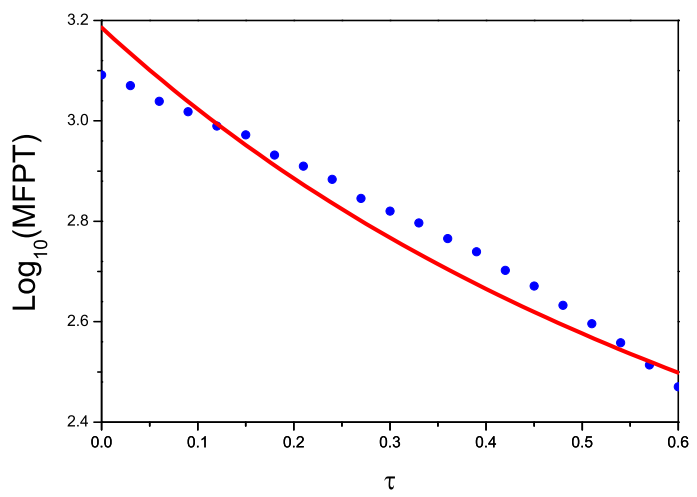

Fig. 7. MFPT is plotted as a function of the delay time $\tau$. $\alpha=0.01, D=0.015$ and $\lambda=0.3$. The other parameter values are the same as those in Fig.3.The red sold line represents the theoretical results, and the blue dot line represents the numerical simulation results.

$\langle x\rangle_{s t}$ is decreased with increasing $\tau$. When $\tau$ is small, the TF-A monomer concentrates on the high concentration state. When $\tau$ is increased, the TF-A monomer concentrates on the low concentration state. Namely, for large $\tau$, it is more easy to be at the "off" state (the low concentration state). The effect is similar to the effect of $\tau$ on SPD shown in Fig.5. It also implicates that the time delay induces the gene transition from the "on" state to the "off" state.

\section{Mean first passage time}

For the delay time-induced switch, we will quantify the effects of delay time on the switch between the two stable steady states. When the system is stochastically bistable, a quantity of interest is the time from one state to the other state, which is often referred to as the first passage time. We consider the mean first passage time (i.e., MFPT). Here the MFPT of the process $x(t)$ to reach the high concentration 
state $x_{+}(\mathrm{t})$ with initial condition $x(t=0)=x_{-}$(the low concentration state) is provided by[38],

$$
T\left(x_{-} \rightarrow x_{+}\right)=\int_{x_{-}}^{x_{+}} \frac{d x}{B(x) P_{s t}(x)} \int_{0}^{x} P_{s t}(y) d y .
$$

When the intensities of noises terms $D$ are small enough compared with the energy barrier height $\triangle \Phi(x)=\Phi\left(x_{+}\right)-$ $\Phi\left(x_{u}\right)$, we can apply the steepest-descent approximation to Eq.(26). Hence $T$ is simplified as following[39]

$$
T\left(x_{+} \rightarrow x_{u}\right) \approx \frac{2 \pi}{\sqrt{\left|U_{0}^{\prime \prime}\left(x_{+}\right) U_{0}^{\prime \prime}\left(x_{u}\right)\right|}} \exp \left[\frac{\Phi\left(x_{u}\right)-\Phi\left(x_{+}\right)}{D}\right] .
$$

Here, the potential $U_{0}(x)$ is given by Eq.(3) and $\Phi(x)$ is given by Eq.(22). By virtue of Eq.(27), the effects of $\tau$ on the MFPT

can be analyzed. MFPT as a function of $\tau$ is plotted in Fig.7. It shows that MFPT decreases monotonously as $\tau$ increases. From the view point of physics, it means that the delay time can speed up the transition between the two steady states (low concentration state and high concentration state). Namely, the delay time $\tau$ can accelerate the transition of gene switch from "on" state to "off" state.

\section{EFFECTS OF TIME DELAY ON STOCHASTIC RESONANCE}

In the gene transcriptional regulatory process, the external environmental factors, such as the electromagnetic field on the earth, the solar terms and seasonal variation, are the common features. This means that the transcript of gene should have a periodic form. For simplicity, a cosinoidal form $A \cos (\Omega t)$ is adopted to model the external periodic stimulus. The model is shown in Fig.8. If considering the effect of noises, the delay time and the periodic signal, we can rewrite Eq.(9) as following

$$
\begin{aligned}
\frac{d x(t)}{d t}= & \frac{k_{f} x^{2}(t)}{x^{2}(t)+K_{d}}-k_{d} x(t-\tau) \\
& +R_{\text {bas }}-x(t-\tau) \xi(t)+\eta(t)+A \cos (\Omega t),
\end{aligned}
$$

where $\xi(t)$ and $\eta(t)$ are the Gaussian white noise, and their statistical properties are given by Eqs.(5)-(8). $A$ is the amplitude of input periodic signal, $\Omega$ is the frequency, and $\tau$ is the delay time.

Making use of the small delay time approximation of the probability density approach and the stochastic equivalence method, the approximated delay Fokker-Planck equation of this model is given by

$$
\begin{aligned}
\frac{\partial P(x, t)}{\partial t}= & -\frac{\partial}{\partial x}\left[\left((1+\tau)\left(\frac{k_{f} x^{2}}{x^{2}+K_{d}}-k_{d} x+R_{b a s}\right)\right.\right. \\
& \left.+A \cos (\Omega t)+D(1+\tau)^{2} x\right) \\
& -\lambda \sqrt{D \alpha}(1+\tau)) P(x, t)] \\
& +\frac{\partial^{2}}{\partial x^{2}}\left[\left(D(1+\tau)^{2} x^{2}\right)\right. \\
& -2 \lambda \sqrt{D \alpha}(1+\tau) x+\alpha) P(x, t)]
\end{aligned}
$$

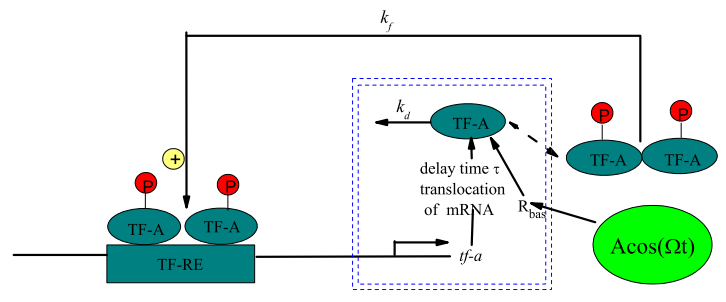

Fig. 8. Model of genetic regulation with a positive autoregulatory feedback loop, delay time and an additive signal $A \cos (\Omega t)$.

Under the constraint $x>0$ (the TF-A monomer concentration $x(t)$ is always higher than zero in the bistable region satisfying Eq.(2), the quasi-steady-state distribution function $P_{q s t}(x, t)$ can be derived from Eq.(29) in the adiabatic limit:

$$
\begin{aligned}
P_{q s t}(x, t)= & \frac{N}{\left(D(1+\tau)^{2} x^{2}-2 \lambda \sqrt{D \alpha}(1+\tau) x+\alpha\right)^{1 / 2}} \\
& \times \exp \left[-\frac{\phi_{n}(x, t)}{D}\right],
\end{aligned}
$$

where $N$ is a normalization constant, $\phi_{n}(x, t)$ is the generalized potential function with the form as below

$$
\begin{aligned}
\phi_{n}(x, t)= & -\frac{D}{\gamma_{0}}\left[\gamma_{1} \ln \left(x^{2}+K_{d}\right)+\gamma_{2} \arctan \left(\frac{x}{\sqrt{K_{d}}}\right)\right. \\
& +\gamma_{3} \ln \left(D n^{2} x^{2}-2 m n x+\alpha\right) \\
& +\frac{\gamma_{4}}{\sqrt{D \alpha n^{2}-m^{2} n^{2}}} \arctan \left(\frac{D n^{2} x-m n}{\sqrt{D \alpha n^{2}-m^{2} n^{2}}}\right) \\
& +\frac{\gamma_{5}}{\sqrt{D \alpha n^{2}-m^{2} n^{2}}} \\
& \left.\times \arctan \left(\frac{D n^{2} x-m n}{\sqrt{D \alpha n^{2}-m^{2} n^{2}}}\right) A \cos (\Omega t)\right]
\end{aligned}
$$

where $n, m, \gamma_{0}, \gamma_{1}, \gamma_{2}, \gamma_{3}$ and $\gamma_{4}$ are given by Eq.(23). And

$$
\gamma_{5}=-2 \alpha K_{d} D n^{3}+n \alpha^{2}+4 K_{d} n^{3} m^{2}+K_{d} n^{5} D^{2} .
$$

Since the frequency $\Omega$ is very small, there is enough time for the system to reach the local equilibrium during the period of $1 / \Omega$. On the other hand, assuming that the amplitude of input periodic signal is enough small $(A<<1)$, it can not make the particles transit from a well to another well. Using the definition of mean first-passage time and steepest descent method, one can obtain the expression of transition rates $W_{ \pm}$ out of $x_{+}, x_{-}$,

$$
\begin{aligned}
& W_{+}=\frac{\sqrt{\left|U_{0}^{\prime \prime}\left(x_{+}\right) U_{0}^{\prime \prime}\left(x_{u}\right)\right|}}{2 \pi} \exp \left[\frac{\phi_{n}\left(x_{+}, t\right)-\phi_{n}\left(x_{u}, t\right)}{D}\right] \\
& W_{-}=\frac{\sqrt{\left|U_{0}^{\prime \prime}\left(x_{-}\right) U_{0}^{\prime \prime}\left(x_{u}\right)\right|}}{2 \pi} \exp \left[\frac{\phi_{n}\left(x_{-}, t\right)-\phi_{n}\left(x_{u}, t\right)}{D}\right] .
\end{aligned}
$$

in which $U(x), x_{+}, x_{-}, x_{u}$ and $\phi_{n}(x, t)$ are defined by Eq.(3) and Eq.(31), respectively.

For the general asymmetric nonlinear dynamical system, the SR phenomenon has been found, and the related theory has 
been developed[40]. Here, we only simply list this method for calculating signal to noise ratio (SNR).

The system is subjected to a time dependent signal $A \cos (\Omega t)$, up to first order on its amplitude (assumed to be small) the transition rates can be expanded as follows by twostate model theory:

$$
\begin{aligned}
& W_{+}=\mu_{1}-\beta_{1} A \cos (\Omega t), \\
& W_{-}=\mu_{2}+\beta_{2} A \cos (\Omega t) .
\end{aligned}
$$

where the constants $\mu_{1,2}$ and $\beta_{1,2}$ depend on the detailed structure of the system under study. For the asymmetric case, $\mu_{1} \neq \mu_{2}$ and $\beta_{1} \neq \beta_{2}$.

For the general asymmetric case we defined $R_{S N R}$, the SNR, as the ratio of the strength of the output signal and the broadband noise output evaluated at the signal frequency. Finally, the expression of SNR is given by[40]

$$
R_{S N R}=\frac{A^{2} \pi\left(\mu_{1} \beta_{2}+\mu_{2} \beta_{1}\right)^{2}}{4 \mu_{1} \mu_{2}\left(\mu_{1}+\mu_{2}\right)},
$$

where

$$
\begin{aligned}
& \mu_{1}=\left.W_{+}\right|_{A \cos (\Omega t)=0}, \\
& \mu_{2}=\left.W_{-}\right|_{A \cos (\Omega t)=0,}, \\
& \beta_{1}=\left.\frac{d W_{+}}{d(A \cos (\omega t))}\right|_{A \cos (\Omega t)=0}, \\
& \beta_{2}=\left.\frac{d W_{-}}{d(A \cos (\omega t))}\right|_{A \cos (\Omega t)=0},
\end{aligned}
$$

According to the expression of SNR in Eq.(36), the effects of the additive noise intensity $\alpha$, the correlated noise intensity $\lambda$ and the delay time $\tau$ on the SNR are analyzed. These results are plotted in Figs.9-11. In Fig.9-11, there exist one or two peaks which is the identifying characteristic of the SR phenomenon. It implicates that the SR happens in this genetic regulatory system.

The SNR as a function of multiplicative noise intensity $D$ with different delay time $\tau=0.1,0.3,0.4$ is plotted in Fig.9a (the other parameters are fixed). It is found that there is a single peak in $R_{S N R}$ vs. $D$. The height of the peak is increased slightly as the delay time $\tau$ increases, and the position of the peak shifts from the large $D$ to small $D$. It implicates that the $R_{S N R}$ is enhanced with the increasement of delay time $\tau$. It must be pointed out that the observed SR is obvious when the additive noise intensity $\alpha$ and the multiplicative noise intensity $D$ are very weak.

The SNR as a function of the multiplicative noise intensity $D$ with different additive noise intensity $\alpha=0.01,0.03$, 0.05 is plotted in Fig.10a (the other parameters are fixed). Comparing the curve of SNR for $\alpha=0.01$ to the curve of SNR for $\alpha=0.02$, the height of the peak is decreased greatly,
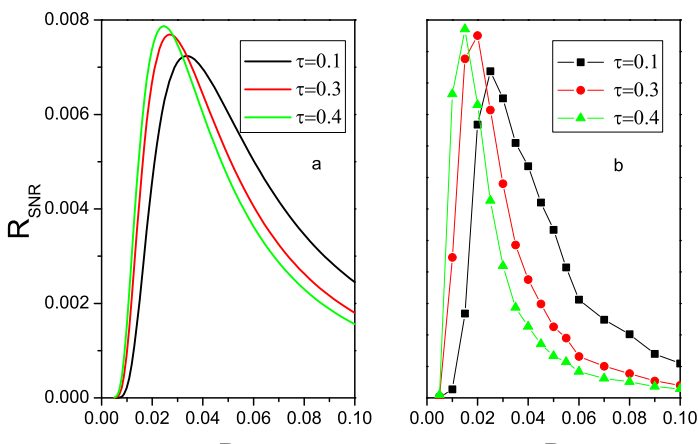

$\mathrm{D}$

D

Fig. 9. $\quad R_{S N R}$ is plotted as the function of multiplicative noise intensity $D$ for different delay time $\tau=0.1,0.3$ and 0.4 with $\alpha=0.01, \lambda=0.5$, $A=0.08$ and $\Omega=0.001$, the other parameter values are the same as those in Fig. 3. (a) Theoretical results (Eq.(31)); (b) numerical simulation results.

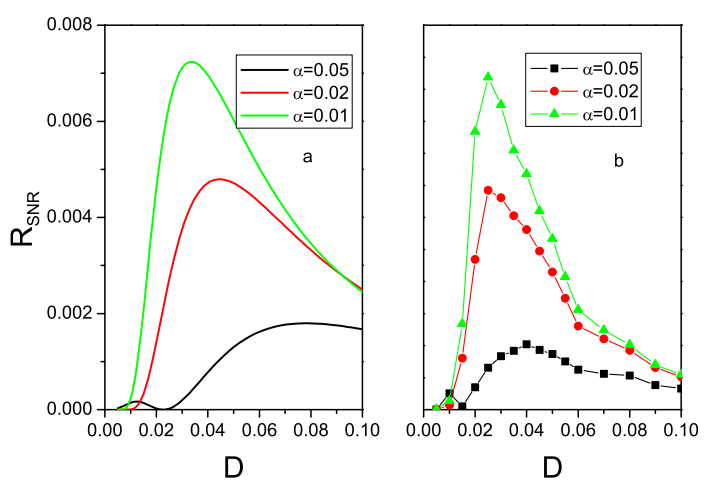

Fig. 10. $R_{S N R}$ is plotted as the function of multiplicative noise intensity $D$ for different additive noise intensity $\alpha=0.01,0.03$ and 0.05 with $\tau=0.1$, $\lambda=0.5, A=0.08$ and $\Omega=0.001$, the other parameter values are the same as those in Fig. 3. (a) Theoretical results (Eq.(31)); (b) numerical simulation results.
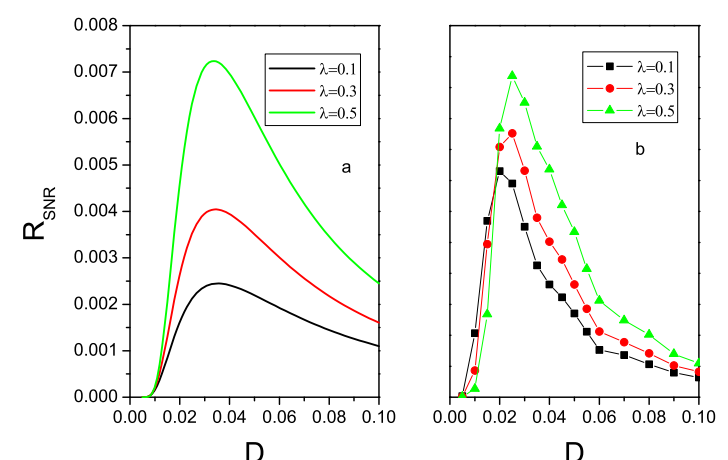

D

Fig. 11. $R_{S N R}$ is plotted as the function of multiplicative noise intensity $D$ for different correlated noise intensity $\lambda=0.1,0.3$ and 0.5 with $\tau=0.1$, $\alpha=0.015, A=0.08$ and $\Omega=0.001$, the other parameter values are the same as those in Fig. 3. (a) theoretical results (Eq.(31)); (b) numerical simulation results. 
and the position shifts slightly from the small value of $D$ to the large value of $D$. Specially, when $\alpha=0.05$, the resonance bi-peaks are found in the curve of SNR. It means that the curve of SNR is changed from one peak to two peaks as $\alpha$ increases. Namely, the additive noise intensity $\alpha$ can restrain the SR and induce the multiple SR. It must be emphasized that the height of the first peak of SNR is more lower than the one of the second peak, and the position of the first peak is located in the very small value of the multiplicative noise intensity $D$.

The SNR as a function of the multiplicative noise intensity $D$ with different correlated noise intensity $\lambda=0.1,0.3,0.5$ is plotted in Figure. 11 a, when the other parameters are fixed. It is seen that the height of the peak is enhanced greatly as the $\lambda$ increases, the positions of the peaks are almost no distinct. It means that the correlated noise intensity $\lambda$ can improve the SR.

Why these different control parameters exhibit various regulatory properties on the SR? One possible reason is that the potential function of the bistable gene model is adjusted differently. The symmetry of potential wells and the height of potential barrier have different dependences on these parameters. The quantitative analysis about the underlying mechanisms of time delay-enhance SR is our next task.

In order to check the valid of the approximate method, the numerical simulation is performed by directly integrating the Eq.(28) with Eqs.(5)-(8). Using the Euler method, the numerical data of time series are calculated using a fast Fourier transform. To reduce the variance of the result, the 1024 ensembles of power spectra are averaged. The output signalto-noise ratio is defined as $R=10 \log 10\left(S_{p}\left(\Omega_{s}\right) / S_{n}\left(\Omega_{s}\right)\right)$, where $S_{p}\left(\Omega_{s}\right)$ is the height of the peak in the power spectrum at the input frequency $\Omega_{s}$ and $S_{n}\left(\Omega_{s}\right)$ is the height of the noisy background in the power spectrum around $\Omega_{s}$. The parameters are chosen as the same value in the theoretical analysis. The results are plotted in Fig.9b, Fig.10b and Fig.11b. Compared Fig.9a, Fig.10a and Fig.11a. to Fig.9b, Fig.10b and Fig.11b, respectively, it is clear that the trends of the approximate theoretical results in the SNR are consistent with the numerical simulation, which implies that the approximate method is credible.

\section{CONClusion}

In this article, the roles of time delay in inducing gene switch and stochastic resonance are systematically studied based on a gene transcriptional regulatory model. We mainly concentrated on two aspects, i.e., the dynamical switch characters (including the steady probability distribution, the mean value and the mean first passage time) and the stochastic resonance phenomenon. Our theoretical results show that (i) the delay time resulting from the transcription of $t f$ - $a$ mRNA and its movement to translation can induce the gene switch process, i.e., the TF-A monomer concentration $x$ shifts from the high concentration state to the low concentration state ("on" $\rightarrow$ "off"). The delay time $\tau$ can also further speed up the transition from "on" to"off". (ii) The stochastic resonance can be enhanced by the time delay $\tau$ and the correlated noise intensity $\lambda$. However, the additive noise original from the synthesis rate $R_{b a s}$ can suppress the stochastic resonance. It is very interesting that the bi-peaks structure is found when $\alpha=0.05$. Through our stochastic delay dynamic approach, the critical physiological control parameters to which the behavior of special genetic regulatory systems is particularly sensitive are identified. Our theoretical results based on smalldelay time-approximation approach are consistent with the numerical simulation, which implies that the approximate method is reliable. These insights on the combined effects of noises and time delay would be beneficial to understanding the basic mechanism of how living systems optimally facilitate to function under real environments.

How could our predictions be related to the experiments? To test our predictions quantitatively, one would ideally like to perform an experiment on this gene transcriptional regulatory model with tunable time delay and other control parameters, in which all the parameters concentrations of components and rate constants are the same as our theoretical model. To our knowledge, this clearly seems a very difficult experiment to perform, what we do is to give a primary picture of the integrated effects of time delay and noise. Recently, with the development of synthetic biology, some artificial gene networks are designed by genetic engineer. Moreover, it is increasingly being recognized that some biological parameters, including time delay and feedback strength, can be controlled by using micro-fluidic devices in gene regulatory network. So we wish that the time delay-accelerated transition of gene switch and time delay-enhanced stochastic resonance could be examined in future.

\section{ACKNOWLEDGMENT}

This project is supported by the Natural Science Foundation of China (Grant No.11047146(CJ Wang), Grant No.10905089 (M Yi) ), the Science Foundation of the Education Bureau of Shanxi Province(Grant No.09JK331(CJ Wang)), the Natural Science Foundation of Shanxi province of China (Grant No. 2010JQ1014(CJ Wang))and the Science Foundation of Baoji University of Science and Arts of China (Grant No.ZK0954(CJ Wang)).

\section{APPENDICES}

In this section, we consider the delay effect in the Hill function. the delay effect on the Hill function in Eq. (4) is considered in this section. Then, $\frac{k_{f} x^{2}(t)}{x^{2}(t)+K_{d}} \rightarrow \frac{k_{f} x^{2}(t-\tau)}{x^{2}(t-\tau)+K_{d}}$, and Eq. (4) can be rewritten:

$$
\frac{d x(t)}{d t}=\frac{k_{f} x^{2}(t-\tau)}{x^{2}(t-\tau)+K_{d}}-k_{d} x(t)+R_{b a s}-x(t) \xi(t)+\eta(t),
$$

where the first term on the right side is evaluated at a time $\tau$ (delay time) previous to the time when $d x / d t$ is computed, 


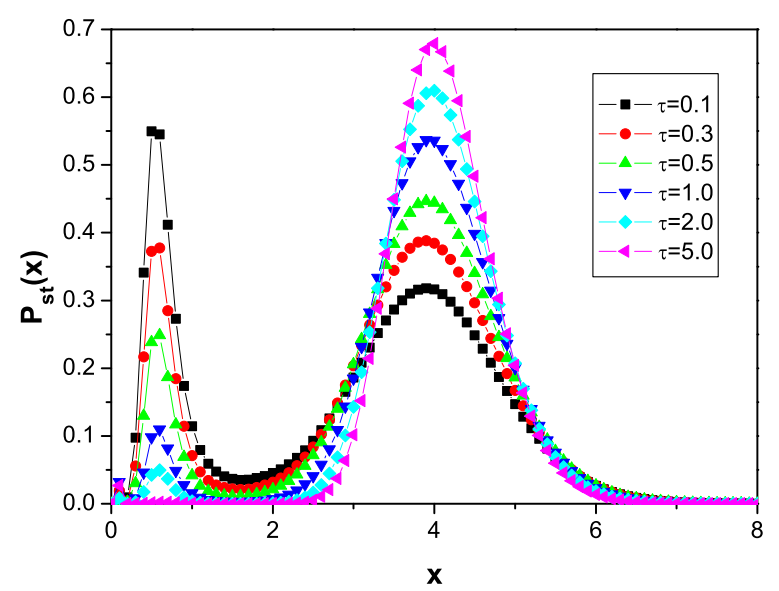

Fig. 12. The numerical simulations of the probability distribution $P_{s t}(x)$ are plotted with the different delay time $\tau=0.1,0.3,0.5,1,2,5$ with $\alpha=0.005$, $D=0.015$, and $\lambda=0.3$

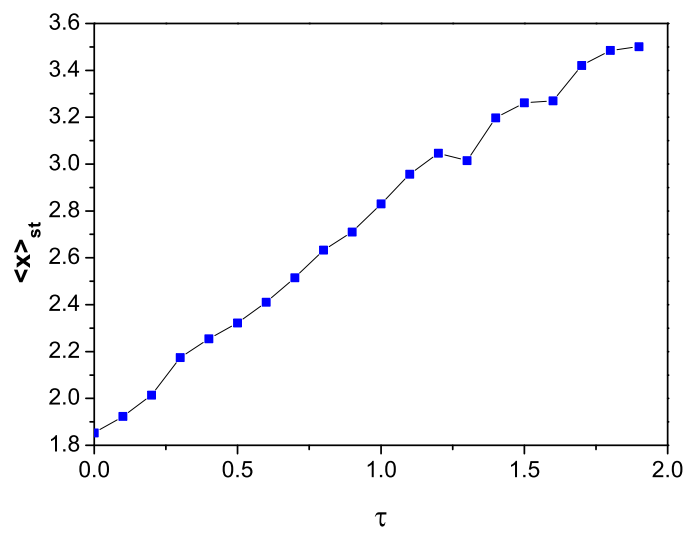

Fig. 13. The numerical results of the mean value of $x(t)$ )for this system as a function of large $\tau_{2}$ are plotted with $\alpha=0.005, D=0.03, \lambda=0.3$ in the nonlinear delay case. The other parameters are the same as those in Fig.3.

and is nonlinear time-delayed, and the delay time does not appear in the stochastic fore.

For the sake of the difficult to deal with from the aspect of the theory, here the following results are given by directly simulating the stochastic delay differential Eq. (A.1), and it can be formally integrated by using a simple forward Eular algorithm with a small time step for small and large delay time.

Figure.12 shows the SPD as a function of the TF-A monomer concentration $x$ for the delay time $\tau$. This result shows that the TF-A monomer concentration $x$ shifts from the low concentration state to the high concentration state with the delay time $\tau$ increasing

The numerical results of the mean value of $x(t)$ )for this

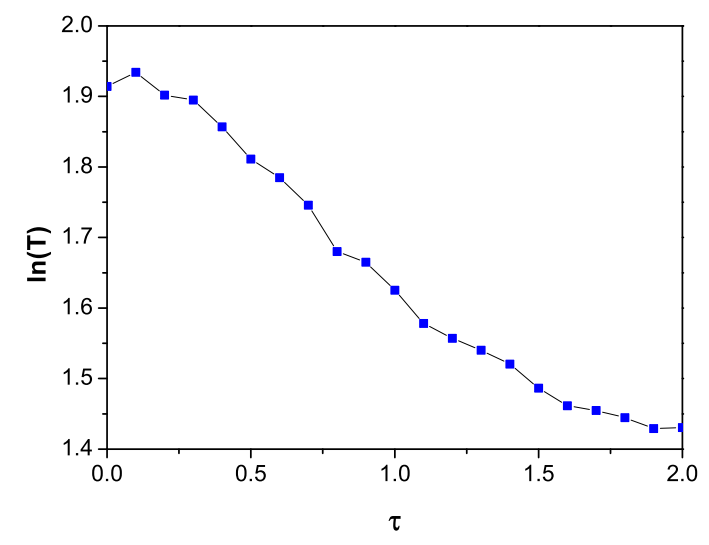

Fig. 14. The numerical results of the mean first passage time (MFPT)for this system with the initial condition $x_{-}$are plotted as a function of large $\tau_{2}$ with $\alpha=0.005, D=0.03, \lambda=0.3$ in the nonlinear delay case. The other parameters are the same as those in Fig.3.

system as a function of $\tau$ are plotted in Fig.13. The result presents the mean value of $x(t))$ increases with $\tau$ increasing.

Figure 14 shows the MFPT of the gene transcriptional regulatory system for the delay time in the nonlinear delay case. It shows that there exists a resonance restrain phenomenon for the small $\tau$. Namely, the small delay time region the MFPT behaves as one maximum. The peak of the MFPT actually corresponds to the suppression plateau in the curve of $\nu-\tau$ (escape rate $\nu$, defined as $1 / T$ ). It implies that the delay time $\tau_{2}$ can restrain the transition from "off" state to "on" state. It must emphasized that the MFPT decreases monotonously with $\tau$ increasing and there is no extremum, when the delay time is large. Namely, in the large delay time region the delay time $\tau_{2}$ can speeds up the transition from "off" state to "on" state.

\section{REFERENCES}

[1] W. Horsthemke and R. Lefever, "Noise-Induced Transitions: Theory and Applications in Physics, Chemistry and Biology," Berlin: SpringerVerlag, 1984.

[2] D. J. Wu, L. Cao, S. Z. Ke, "Bistable kinetic model driven by correlated noises: Steady-state analysis," Phys. Rev. E ,Vol. 50, 1994, pp. 24962502.

[3] F. Moss and P. V. E. McClintock, "Noise in Nonlinear Dynamical Systems, Vols. I and II, Cambridge, Cambridge University Press, 1989.

[4] F. Castro, A. D. Sánchez and H. S. Wio, "Reentrance Phenomena in Noise Induced Transitions," Phys. Rev. Lett., vol. 75, 1995, PP. 16911694.

[5] Y. Jia, J.R. Li, "Reentrance Phenomena in a Bistable Kinetic Model Driven by Correlated Noise,” Phys. Rev. Lett.,Vol.78,1997, pp. 994-997.

[6] L. Gammaitoni, P. Hänggi, P. Jung, F. Marchesoni, "stochastic resonance,"Rev. Mod. Phys. Vol. 70, 1998,pp. 223-287.

[7] J. K. Douglass, L. Wilkens, E. Pantazelou, F. Moss, "Noise enhancement of information transfer in crayfish mechanoreceptors by stochastic resonance," Nature, Vol.365, 1993, pp. 337-340.

[8] R. N. Mantegna and B. Spagnolo, "Noise Enhanced Stability in an Unstable System," Phys. Rev. Lett. Vol.76, 1996, pp.563-566.

[9] A.A. Dubkov, N. V. Agudov, B. Spagnolo, "Noise-enhanced stability in fluctuating metastable states," Phys. Rev. E, Vol.69, 2004,pp. 061103 
[10] L. Cao and D. J. Wu, "Fluctuation-induced transport in a spatially symmetric periodic potential," Phys. Rev. E, VO1. 62, 2004, pp.74787481.

[11] J. H.Li, J. Lczka and P. Hänggi, "Transport of particles for a spatially periodic stochastic system with correlated noises," Phys. Rev. E, VOl. 64, 2001, pp. 011113

[12] C.J. Wang, S.B. Chen and D.C. Mei, "Current reversal in a continuously periodic system driven by an additive noise and a multiplicative noise," Phys. Lett. A , Vol.352, 2006, pp.119-123.

[13] C. Masoller, "Noise-induced resonance in delayed feedback system," Phys. Rev. Lett. Vol.88,2002,pp.034102.

[14] D.Huber and L. S. Tsimring,"Dynamics of an ensemble of noisy bistable elements with global time delayed coupling," Phys. Rev. Lett. , Vol.91, 2003,pp. 260601.

[15] L. S. Tsimring, A. Pikovsky, "Noise-induced dynamics in bistable systems with delay," Phys. Rev. Lett. , Vol.87, 2001, pp.250602.

[16] D. Wu and S.Q. Zhu, "Brownian motor with time-delayed feedback," Phys. Rev. E, Vol. 73, 2006, pp.051107.

[17] E. M. Craig ,B. R. Long , J. M. R. Parrondo and H. Linke,"Effect of time delay on feedback control of a flashing ratchet," Eur. Phys. Lett. , Vol. 81, pp. 10002.

[18] L. R. Nie and D. C. Mei, "Noise and time delay: Suppressed population explosion of the mutualism system," Eur. Phys. Lett., Vol. 79, 2007,pp. 20005 .

[19] L. R. Nie and D. C. Mei, "Effects of time delay on symmetric twospecies competition subject to noise," Phys. Rev. E, Vol. 77,2008, pp.031107.

[20] P. Smolen, D. A. Baxter, J.H. Byrne, "Frequency selectivity, multistabil ity, and oscillations emerge from models of genetic regulatory systems,' Am. J. Physiol., Vol.274, 1998, pp.C531.

[21] P. Smolen, D. A. Baxter, J.H. Byrne,"Effects of macromolecular transport and stochastic fluctuations on dynamics of genetic regulatory systems," Am. J. Physiol.,Vol.277, 1999, pp. C777.

[22] J. Hasty, M. Dolnik, V. Rottschafer, and J. J. Collins, "Synthetic Gene Network for Entraining and Amplifying Cellular Oscillations," Phys. Rev. Lett., Vol.88, 2002, pp.148101.

[23] Q. Liu and Y. Jia, "Fluctuations-induced switch in the gene transcriptional regulatory system," Phys. Rev. E , Vol. 70,2004, pp. 041907.

[24] C. J. Wang, D. C. Mei, "Effect of colored cross-correlated noise on the in the gene transcriptional regulatory system," ACTA PHYSICA SINICA, Vol.57, pp. 3983.

[25] J. Y. Wang, C. L. Zhu, Y. Jia, and J. R. Li "Effects of nonlinear timedelay on a stochastic asymmetric System," Chin. Phys. Lett., Vol.23, 2007,pp. 1398

[26] J. W. Wang , J. J.Zhang , Z. J. Yuan and T. S.Zhou, "Noise-induced switches in network systems of the genetic toggle switch,"BMC systems biology, Vol.1, 2007, pp.50.

[27] R. Benzi, A. Sutera, and A. Vulpiani," Stochastic resonance in climatic change," Tellus, Vol.34, 1982, pp.10-16.

[28] C. Nicolis ,G. Nicolis, "Stochastic aspects of climatic transitionsresponse to a periodic forcing,'Tellus, Vol.34, 1982, pp. 1-9.

[29] E.Simonotto,M. Riani , C. Seife , M.Roberts, J. Twitty, and F. Moss, "Visual Perception of Stochastic Resonance," Phys. Rev. Lett., Vol. 78, 199, pp. 1186-1189.

[30] W. R. Zhong , Y. Z.Shao, and Z. H.He, "Pure multiplicative stochastic resonance of a theoretical anti-tumor model with seasonal modulability," Phys. Rev. E, Vol. 73, 2006, pp. 060902(R).

[31] H. A. Braun , H.Wissing, K. Schafer , and M. C. Hirsch, "Oscillation and noise determine signal transduction in shark multimodal sensory cells,", Nature, Vol.367, 1994,pp. 270.

[32] M. Thattai and A. van Oudenaarden,"Intrinsic noise in gene regulatory networks, " Proc. Natl. Acad. Sci. U.S.A., Vol.98, 2001,pp. 8614.

[33] G. van Dassow , G.Meir , E. M. Munro, and G. M.Odell,"The segment polarity network is a robust developmental module," Nature (London), Vol.406, 2000, pp.188 -192.

[34] K. Ahmad and S. Henikoff, "Modulation of a Transcription Factor Counteracts Heterochromatic Gene Silencing in Drosophila," Cell, Vol. 104, 2001,pp. 839-847.

[35] T. D. Frank, "Delay Fokker-Planck equations, perturbation theory, and data analysis for nonlinear stochastic systems with time delays," Phys. Rev. E, Vol.71, 2005, pp. 011112.

[36] X. Gu, S. Zhu, and D. Wu, "Two different kinds of time delays in a stochastic system," Eur Phys. J. D, Vol. 42, 2007,pp. 461-466.
[37] H. Risken, "The Fokker-Planck Equation: Methods of Solution and Applications," Berlin: Springer-Verlag, 1989.

[38] J. Masoliver, B.J. West, K. Lindenbergerg,"Bistability driven by Gaussian colored noise: First-passage times," Phys. Rev. A, Vol.35, 1987,pp. 3086-3094.

[39] R. F.Fox, "Functional-calculus approach to stochastic differential equations,"Phys. Rev. A, Vol. 33,1986, pp. 467-476.

[40] H. S. Wio and S. Bouzat, "Stochastic resonance: the role of potential asymmetry and non Gaussian noises,'Braz. J. Phys., Vol. 29, 1999, pp. $136-143$. 